A N N A L E S Annales de Bretagne et des Pays de l'Ouest

\title{
Les sociétés littorales face aux pouvoirs au XVIIIe siècle
}

\section{Morgane Vary}

\section{(2) OpenEdition}

\section{Journals}

Édition électronique

URL : http://journals.openedition.org/abpo/905

DOI : 10.4000/abpo.905

ISBN : 978-2-7535-1501-7

ISSN : 2108-6443

Éditeur

Presses universitaires de Rennes

Édition imprimée

Date de publication : 30 mars 2006

Pagination : $71-86$

ISBN : 978-2-7535-0273-4

ISSN : 0399-0826

\section{Référence électronique}

Morgane Vary, «Les sociétés littorales face aux pouvoirs au XVIIle siècle », Annales de Bretagne et des

Pays de l'Ouest [En ligne], 113-1 | 2006, mis en ligne le 30 décembre 2008, consulté le 20 avril 2019.

URL : http://journals.openedition.org/abpo/905 ; DOI : 10.4000/abpo.905 


\title{
Les sociétés littorales face aux pouvoirs au XVIII ${ }^{\mathrm{e}}$ siècle
}

\author{
Morgane VARY \\ ATER en histoire moderne \\ SOLITO - université de Bretagne-Sud
}

Le règne de Louis XIV se caractérise par une volonté de reprise en main de la société passant par la création de nouvelles institutions. Parallèlement, certaines instances judiciaires voient leurs attributions redéfinies. La mise en place de ces juridictions s'est faite non pas par complémentarité, mais plutôt par juxtaposition. À cet égard, le littoral apparaît comme un espace particulièrement surveillé. La population autochtone y côtoie une population migrante et flottante considérée comme dangereuse par les élites. Cet espace d'interface semble favoriser la mobilité que ce soit dans le cadre de micro-déplacements ou de migrations à plus grande échelle.

Nous baserons notre étude sur le littoral de la Bretagne occidentale et méridionale, zone géographique illustrant particulièrement bien cette volonté étatique de surveillance des populations par la mise en place d'un maillage juridictionnel dense. Les ressorts des différentes juridictions présentes sur cet espace se superposent et s'entremêlent dans un souci d'efficacité. Cette juxtaposition provoque une certaine confusion chez les populations littorales, voire même un rejet de certaines institutions. La multiplicité des juridictions peut parfois être un obstacle à leur efficience.

Il convient de décrire brièvement le maillage juridictionnel présent sur le littoral de la Bretagne occidentale et méridionale et de s'interroger sur la réelle efficacité des instances judiciaires sur cet espace en analysant les rapports entretenus entre les institutions et la population.

\section{La superposition des juridictions}

\section{Les justices seigneuriales}

Ces justices représentent la plus infime strate du maillage juridictionnel. Il semble difficile d'établir une description de l'ensemble des justices seigneuriales s'étendant sur le littoral breton d'autant qu'une même paroisse pouvait relever de plusieurs de ces justices de proximité. 
O.-H. Hufton souligne la nécessité d'un tel tribunal : " Pour la famille paysanne qui subit les atteintes constantes à sa propriété, il est nécessaire de défendre chaque brin d'herbe convoité et d'avoir à portée de la main un tribunal ne demandant que peu de frais pour régler les problèmes familiaux, d'héritage ou de transaction des terres, de tutelle, etc. ${ }^{1}$. "

Cependant, la notion de cas royal, s'étendant aux crimes tels que le vol ou encore l'assassinat, va restreindre l'activité criminelle de ce type de juridictions. Ces affaires vont échoir aux juridictions royales ordinaires.

\section{Les juridictions royales ordinaires : présidiaux et sénéchaussées}

Le ressort des présidiaux est très étendu : il comprend le littoral breton mais aussi l'intérieur de la province. En 1552, Henri II établit les présidiaux de Vannes, Nantes, Rennes et Quimper. En 1789, le ressort du présidial de Vannes s'étend sur les sénéchaussées de Vannes, Auray, Belle-Île, Hennebont, Ploërmel, Quimperlé et Rhuys (Figure 1). L'espace circonscrit par cette juridiction étant très étendu, sa proximité avec la population en est moins importante. Les mêmes constatations peuvent être faites concernant les présidiaux de Nantes (Figure 2) et de Quimper (Figure 3).

Le territoire des sénéchaussées est plus restreint, ce qui facilite les rapports entre les agents du roi et la population. Par exemple, la sénéchaussée de Rhuys ne comprend que quelques paroisses (Arzon, Houat et sa trève d'Hoëdic, l'île d'Arz, Saint-Gildas-de-Rhuys et Sarzeau) (Figure 1). Le sénéchal exerce la haute, la moyenne et la basse justice ainsi que l'appel des causes jugées par les sièges inférieurs. Néanmoins, cette juridiction va pâtir de l'essor du présidial. Pour preuve, la sénéchaussée de Nantes a été absorbée peu à peu par le présidial du même nom et le siège de Vannes connaît le même sort. Les autres sénéchaussées doivent faire face à la concurrence des présidiaux.

Ce ne sont pas ces juridictions ordinaires qui confèrent au littoral son caractère original, mais bien les justices d'exception dont l'établissement permet une surveillance accrue de la population vivant sur ce territoire.

\section{Les juridictions royales extraordinaires}

Nous limiterons notre présentation à quatre juridictions : la maréchaussée, l'amirauté, la justice militaire maritime et les traites.

La maréchaussée n'exerce pas spécifiquement ses prérogatives sur le littoral. Elle apparaît au milieu du $\mathrm{XV}^{\mathrm{e}}$ siècle en Bretagne et, au début du $\mathrm{XVI}^{\mathrm{e}}$ siècle, elle a des attributions limitées : elle s'occupe essentiellement de la surveillance des gens de guerre. L'édit de 1554 étend ses compétences

1. Hufton, Olwen-Hazel, "Le paysan et la loi en France au XVIII ${ }^{\mathrm{e}}$ siècle ", Annales Économie, Société et Civilisation, mai-juin 1983, p. 679-701, voir p. 681. 
sur les " non militaires, errants et vagabonds, ou même domiciliés ". Toute personne suspecte peut être susceptible d'être appréhendée. Or, le littoral est un territoire favorisant la mobilité : la population migrante et flottante y est plus nombreuse, ce qui fournit un surcroît de travail aux agents de la maréchaussée.

À la veille de la Révolution, les brigades de la lieutenance de Nantes sont réparties de la manière suivante : deux brigades pour la ville de Nantes, une brigade pour La Roche-Bernard, Redon, Ancenis, Nozay, Machecoul, Paimbœuf, Savenay, Châteaubriant. Le siège de Vannes se compose d'une brigade à Vannes, Pontivy, Ploërmel, Quimperlé, Hennebont et Lorient. La lieutenance de Quimper, quant à elle, comprend une brigade à Quimper, Châteaulin, Landerneau, Brest, Morlaix et Carhaix. Nous pouvons constater que plusieurs villes maritimes sont concernées. Les brigadiers et les cavaliers de maréchaussée sont chargés d'encadrer la population à la fois par leurs tournées dans les campagnes environnantes et par leur surveillance des principaux axes de communication, mais aussi et surtout par leur intrusion dans un nouveau territoire : la ville. En effet, cette extension du domaine d'activité de la maréchaussée va de paire avec une nouvelle conception de la ville moderne ${ }^{2}$. Cependant, d'après l'ordonnance de 1760, ces patrouilles dans la ville doivent conserver un caractère exceptionnel. Elles se soldent cependant souvent par des arrestations nombreuses puisque la ville, et, a fortiori, la ville maritime abrite bon nombre de pauvres hères attirés par les possibilités d'emploi.

La présence de l'amirauté confère au littoral un caractère original. En effet, l'établissement de cette juridiction supplémentaire vise à contrôler la population navigante mais également la population littorale en général : l'amirauté s'occupe de tous les litiges ayant pour théâtre la mer et ses rivages. Au même titre qu'une juridiction ordinaire, l'amirauté a connaissance des affaires de vols, d'assassinats ou encore de mauvais traitements. Le lieu où s'exercent ses prérogatives lui confère néanmoins un caractère spécifique. Ainsi, la majeure partie de son activité consiste à juger des cas de naufrages, de pillages et de barateries.

Après bien des errements, un édit particulier établit, en 1691, sept sièges d'amirautés en Bretagne à Saint-Malo, Saint-Brieuc, Morlaix, Brest, Quimper, Vannes et Nantes. L'amirauté de Léon, dont le siège se trouve à Brest, englobe un espace littoral compris entre la rivière de Morlaix et l'embouchure de l'Élorn également appelée rivière de Landerneau ${ }^{3}$. L'amirauté de Cornouaille, dont le siège se situe à Quimper, a juridiction de la rivière de l'Élorn à la Laïta (ou rivière de Quimperlé). Le siège de Vannes étend son

2. DyONET, Nicole, "La maréchaussée et la ville en France au XVIII ${ }^{\mathrm{e}}$ siècle ", dans : Le pénal dans tous ses États, Justice, États et sociétés en Europe (XII -XXe siècle), RoussEAUX Xavier et LEVy René (dir.), Bruxelles, Publications des Facultés Universitaires Saint Louis, 1997, 462 p., voir p. 323-335.

3. Il est également nécessaire d'y adjoindre la paroisse de Saint Thomas de Landerneau située de l'autre côté de la rivière de Landerneau. 
pouvoir sur un espace allant de la Laïta à l'embouchure de la Vilaine. Enfin, le siège nantais contrôle la côte de la Vilaine jusqu'au marais de Bouin. Il faut aussi mentionner la création de l'amirauté de Lorient par une lettre patente datée du 12 avril 1782. Quatre ans plus tard, le siège de Lorient obtient son indépendance. Cette création est tardive si on se réfère aux sollicitations présentées par l'Intendant de Marine Clairambault dès 1709, prétextant que le siège de Vannes est trop éloigné des principaux lieux de dynamisme économique. Le ressort de Lorient s'étend de la Laïta à la rivière d'Étel.

La justice militaire maritime, une autre justice d'exception, vient restreindre les attributions de l'amirauté. En effet, l'amirauté ne peut juger les gens de mer lorsqu'ils sont en activité pour le service du roi. L'ordonnance de la Marine d'août 1681 précise que la compétence de cette juridiction " ne s'étend pas aux délits commis sur les Vaisseaux du Roi ou dans ses Ports, par les Officiers ou Soldats de la Marine. C'est au conseil de Guerre de chaque département d'en connoitre précisément [...] c'est à ce conseil de Guerre tout de même que les déserteurs doivent être jugés et punis ${ }^{4}$ ". Cependant, les vols commis dans l'arsenal sont du ressort du tribunal prévôtal de l'intendant de la marine. Par contre, le conseil de guerre devient compétent pour tous les autres crimes et délits commis par quelque personne que ce soit dans l'enceinte de l'arsenal.

Les traites viennent se superposer sur ce canevas juridictionnel déjà bien surchargé. En Bretagne, il existe quatorze juridictions des traites dont la plus ancienne semble avoir été celle de Nantes. Comme le remarque Élisabeth Rogani, " mis à part le siège de la Chambre des Comptes et de l'Intendance, et Rennes, capitale politique de la Bretagne du fait de la présence du Parlement, les autres villes qui ont été par intermittence siège d'une direction provinciale des Fermes bénéficient d'une situation géographique littorale. Il s'agit de Saint-Malo surtout, de la ville du Port-Louis puis de Lorient, port de la Compagnie des Indes ${ }^{5}$ ". Cette juridiction s'occupe du règlement des contestations et des délits relatifs à la perception des droits levés sur les denrées et les marchandises. Ce sont essentiellement des cas de contrebande de tabac qui sont retrouvés dans les archives, mais également des affaires relatives à de fausses déclarations concernant les cargaisons des navires.

\section{Des juridictions efficaces?}

Cet enchevêtrement de juridictions sur le littoral sud breton à la fin du XVIII ${ }^{\mathrm{e}}$ siècle laisse déjà présager plusieurs problèmes : comment ces juri-

4. Cité par Berbouche, Alain, « La justice militaire portuaire à la fin de l'Ancien Régime. Des justices royales d'exception en difficultés ", dans LE BouEDEc, Gérard et ChAPPE, François (dir.), Pouvoirs et littoraux $d u X^{e}$ au $X X^{e}$ siècle, actes du colloque international de Lorient, 24, 25 et 26 septembre 1998, Rennes, PUR, 2000, p. 339-346, voir p. 340.

5. Rogani, Élisabeth, Les Traites entre la Bretagne et le royaume de France de 1664 à 1791, mémoire de DEA sous la direction de Claude NIERES, université Rennes II, septembre 1996, 242 p., voir p. 43. 
dictions s'entendent-elles entre elles, sont-elles efficaces, c'est-à-dire remplissent-elles les objectifs fixés par l'État à savoir le contrôle de la population et l'imposition de certaines normes et valeurs par un processus d'acculturation?

\section{Des conflits d'attribution}

Les conflits d'attribution tendent à ralentir considérablement la procédure judiciaire et par conséquent à rallonger le "séjour " du suspect en prison. Ainsi, la maréchaussée doit subir la tutelle du présidial (sauf pour les cas de vagabondage et de mendicité qui sont assimilables à des procédures extra-judiciaires réglées en trois ou quatre jours). Pour les autres cas (vols, assassinats...), les prévôts doivent endurer l'intrusion des juridictions ordinaires dans leurs affaires. L'ordonnance criminelle de 1670 prévoit qu'un jugement de compétence soit rendu par le présidial dans les trois jours suivant la capture. Mais Joël Hautebert précise que la compétence n'est souvent attribuée qu'au bout de quelques semaines voire plusieurs mois ${ }^{6}$. De plus, les absences du lieutenant du prévôt viennent parfois alourdir la procédure.

Les autres juridictions n'ont pas meilleure réputation. Certaines affaires perdurent pendant quatre, cinq voire six ans suivant les aléas de la procédure, les passages de juridictions en juridictions, l'empressement du juge à régler l'affaire.

\section{Des juridictions récentes}

L'amirauté peine à faire reconnaître sa légitimité, en particulier auprès de juridictions plus anciennes qui voient d'un mauvais œil cette nouvelle création royale. Ce n'est qu'en avril et juin 1691 que les sièges de Nantes et Vannes se passent de la tutelle du présidial et obtiennent leur indépendance. Le caractère récent de cette juridiction ne va pas sans poser de problèmes. En effet, elle intervient dans les cas de naufrages, qu'ils soient suivis ou non de pillages. Si pillage il y a, l'amirauté a fort à faire avec les justices seigneuriales qui voient leurs attributions réduites par l'intrusion de cette nouvelle juridiction. Parfois, des heurts violents opposent ces deux instances.

En effet, le droit de bris échappe au roi, tout au moins jusqu'en 1600. Auparavant, les anciennes Coutumes de Bretagne, Normandie ou encore Poitou accordaient aux seigneuries maritimes " tout ce que l'eau aura bouté et jeté à la mer ${ }^{7}$ ". L'ordonnance de la Marine de 1681 dont le titre IX est consacré aux « Naufrages et Échouements » met en relief cette préoccupa-

6. HAUTEBERT, Joël, La jurisprudence criminelle de la sénéchaussée présidiale de Nantes (1551-1750), thèse de doctorat soutenue le 10 janvier 1996, Faculté de Droit et de Sciences Politiques, Université de Rennes 1, 2 volumes, 501 p., voir p. 101.

7. Cabantous, Alain, Les côtes barbares. Pilleurs d'épaves et sociétés littorales en France, 1680-1830, Paris, Fayard, 1993, 311 p., voir p. 122. 
tion du souverain. Autre coup de boutoir aux prétentions seigneuriales, Louis XIV met « fin au régime particulier de la Bretagne en retirant, en 1684, aux justices seigneuriales le droit de poursuite dans les affaires de pillage, au bénéfice des justices royales ${ }^{8}$ " et, à partir de 1691, ces affaires sont confiées aux amirautés. Cependant, certains seigneurs ignorent délibérément les ordonnances. Selon Alain Cabantous, ces abus vont toutefois régresser sensiblement après $1737^{9}$.

Les abbayes posent également problème à cette jeune juridiction (abbayes de Saint-Gildas-de-Rhuys, abbaye de Prière en Billiers, abbaye Saint Sauveur de Redon, abbaye de la Joie à Hennebont). Celles-ci représentent de véritables poches de résistance, ce qui incite les populations locales à l'indocilité. On y découvre des filets interdits, avec des mailles trop petites ${ }^{10}$, utilisés pourtant depuis des années. De même, l'abbaye de Saint-Gildas-de-Rhuys, qui a sous sa coupe les îles de Houat et Hoëdic, semble tant impressionner les agents de l'amirauté que ceux-ci ne se rendront pas dans ces deux lieux ${ }^{11}$.

L'amirauté connaît également quelques difficultés avec les sénéchaussées :

"En 1783, le parlement de Bretagne prend un arrêt suite aux démêlés des juges de l'amirauté de Cornouaille avec le sénéchal de Concarneau. En effet, celui-ci refuse de reconnaître la compétence de l'amirauté dans sa ville. Il exige d'être informé de tout acte juridictionnel qui y sera fait. Il se rend au greffe d'amirauté et consulte les registres [...]. Il va même jusqu'à faire emprisonner un charretier qui travaille au sauvetage d'effets de naufragés, sur ordre de l'amirauté, au prétexte qu'il contrevient aux règlements un jour de fête chômée ${ }^{12}$."

Finalement, le parlement tranche en faveur de l'amirauté. Toutefois, cela démontre que même à la fin du XVIII ${ }^{\mathrm{e}}$ siècle, les conflits entre nouvelles et anciennes juridictions perdurent.

\section{Des juridictions trop pesantes}

À partir d'un certain degré de pression sur les populations, ces dernières cherchent des moyens détournés (légaux ou illégaux) pour contourner la loi. Lorient est un très bon observatoire de ce type de comportements. Au départ, la ville relève de la haute justice des Rohan-Guémené. En 1786, le prince de Rohan-Guémené vend sa seigneurie et sa juridiction au roi. En 1782, le siège de l'amirauté vient s'y installer. La justice militaire y exerce aussi son office dans l'enceinte de l'arsenal. Enfin, le dédouane-

8. Ibidem, p 127.

9. Ibid., p 128.

10. Arch. dép. du Morbihan, 9B237 et 238 cité par LE Goff, Samuel, L'amirauté de Vannes (1691-1792). Un tribunal et ses officiers, mémoire de DEA, sous la direction d'André LeSPAGNOL, Université de Rennes 2, juin 1998, 141 p., voir p. 37.

11. Le Goff, Samuel, L'amirauté de Vannes..., op. cit., p. 38.

12. Ibidem, p 41. 
ment du port de Lorient concernant le trafic du tabac va accroître la surveillance exercée par les employés des Fermes aux portes de la ville, le long des quais, aux abords des navires...

Cela entraîne, chez les populations, une certaine confusion et surtout un sentiment d'oppression. Elles tentent par tous les moyens de continuer à exercer leurs activités qu'elles soient licites ou illicites. Elles font preuve d'originalité ou usent de violence pour passer entre les mailles du filet. Prenons le cas de Catherine Guillemoto qui est à la tête d'un réseau d'au moins vingt et une personnes dont plus de la moitié travaille dans les entrepôts de la Compagnie des Indes. Pour ce groupe, il s'agit de sortir de ces entrepôts, des cauris, sans éveiller les soupçons des gardiens. Le stratagème est simple mais long. Chaque individu doit cacher au fond de ses poches ou dans ses chaussures une infime quantité de marchandises. En 1743, lorsque le réseau est découvert, Catherine Guillemoto et ses comparses ont collecté pas moins de 920 livres de cauris répartis en 9 sacs cachés au fond du puits de notre infortunée. Nous ne savons pas à qui était destiné le produit du larcin, mais nous pouvons vraisemblablement penser qu'une filière parallèle mettait en rapport ce réseau de voleurs avec un ou plusieurs receleurs, sans doute revendeurs à leurs heures et qui sont pléthores dans cette ville.

À Lorient, les juridictions qui se superposent ne semblent pas vouloir collaborer : ainsi, il semble facile de passer entre les mailles du filet. En effet, même si la pression sur les populations est importante, les esprits ingénieux sont mis à profit pour trouver la moindre faille dans cet imbroglio juridique et certains individus réussissent à commettre leurs méfaits et à fuir sans être inquiétés, ce qui met en cause l'efficacité de ces juridictions et surtout leur manque de coordination. C'est ce qu'on observe dans l'affaire du vol d'une balle de thé par le nommé Kerdrain, commis des Fermes chargé de la balance dans le magasin des ventes à Lorient. Le receleur est connu et pourtant il semble qu'aucune vérification n'ait été faite. Peut-être que la justice militaire portuaire qui semble s'occuper de l'affaire n'a pas compétence à appréhender le fripier. Une coopération entre les différentes juridictions ne semble pas avoir été envisagée.

De même, les populations n'hésitent pas à consommer et surtout faire consommer de l'alcool chez elles, en toute illégalité, transformant leur arrière boutique ou leur chambre en cabaret. Ces comportements existent également à Brest où il semble que les employés aux Devoirs ne fassent guère peur aux habitants. De plus, des stratagèmes sont employés pour cacher les barriques ou pour les transporter d'une maison à l'autre, par des portes dérobées.

\section{Les populations littorales face aux pouvoirs}

Il s'agit de voir comment les populations perçoivent l'intrusion dans leur vie quotidienne des agents de l'État. Sont-elles aussi ignorantes des rouages de la machine judiciaire ou les utilisent-elles au gré de leurs 
besoins. Ces populations sont-elles tantôt frondeuses et rebelles face à l'autorité et tantôt parties prenantes dans la procédure judiciaire en tant que partie plaignante? Existe-t-il des distinctions selon les juridictions : certaines sont-elles mieux acceptées que d'autres et que leur vaut ce statut?

\section{Les réactions des populations littorales face aux représentants de l'autorité royale : de la coopération à la révolte}

L'éventail des réactions des populations littorales face aux représentants de l'autorité royale est large : il s'étend de la franche collaboration, voire de la collusion, à la résistance la plus acharnée face à cette intrusion de l'État dans le quotidien des communautés. Il est difficile de déceler les éventuelles complicités existant entre les agents royaux et la population : elles ne transparaissent que lorsque les coupables sont démasqués. Tel a été par exemple le cas du nommé Kerdrain commis des Fermes chargé de la balance dans le magasin des ventes à Lorient dont nous avons parlé précédemment et dont le vol a permis de mettre au jour son étroite collaboration avec un fripier de la ville.

Il semble nécessaire de s'interroger sur l'origine géographique du personnel judiciaire constamment en contact avec la population. Les différentes études menées sur le sujet démontrent une relative proximité de ces agents. En effet, en grande majorité, ils sont d'origine bretonne en ce qui concerne le personnel des Fermes. Cependant, ils ne sont pas forcément natifs du diocèse dans lequel ils exercent leurs fonctions. Cette proportion de bretons s'explique sans doute par le barrage de la langue : il est préférable de choisir des agents sur le terrain capables de comprendre la langue bretonne, unique idiome maîtrisé par la population pour s'exprimer dans certains cas.

Par contre, on note une plus grande diversité des origines géographiques du personnel de la maréchaussée. Dans la lieutenance de Vannes, seuls douze cavaliers sur trente-six sont originaires de Bretagne ${ }^{13}$. De même, Éric Hestault rappelle que " moins d'un homme sur cinq (18\%) qui servit dans la lieutenance de Nantes entre 1770 et 1789 en était originaire ${ }^{14}$ ". La Bretagne apparaît toutefois comme une exception si on se réfère aux résultats obtenus pour les autres généralités : "Dans les années soixante-dix un bon dixième des hommes servaient dans leur ville d'origine et quelque trois quarts des hommes servaient dans leur généralité d'origine ${ }^{15}$. " Clive Emsley explique cette différence par le fait que « cela provenait sans doute du petit

13. Bertin-Mourot, Éliane, Le personnel de la maréchaussée de Bretagne de 1720 à la Révolution, mémoire de DES d'histoire du droit rédigé sous la direction de J.-M. Turlan, Faculté de droit, Université de Rennes, octobre 1965, 91 p + annexes, voir p. 75.

14. Hestault, Éric, La lieutenance de maréchaussée de Nantes (1770-1791), Maisons-Alfort, Service historique de la gendarmerie nationale, 2002, 488 p., voir p. 172.

15. EMSLEY, Clive, " La maréchaussée à la fin de l'Ancien Régime. Note sur la composition du corps ", Revue d'histoire moderne et contemporaine, t. 33, oct-déc 1986, p. 622-644, voir p. 630 . 
nombre de Bretons qui servaient dans l'armée et remplissaient donc les conditions requises pour le service dans la maréchaussée. La Bretagne fournissait les hommes pour le service dans la marine et d0nnait moins de soldats qu'on n'eut pu l'attendre ${ }^{16}$ ". De plus, il évoque des antagonismes profonds existant entre la population et ce corps :

«En 1771, Etienne de Vic concluait son rapport d'inspection en notant que "le paysan breton conserve une haine marquée contre la maréchaussée". C'était un cercle vicieux : plus les paysans bretons détestaient la maréchaussée moins ils avaient envie de servir dans le corps après l'armée; le corps en apparaissait d'autant plus étranger ce qui accroissait l'hostilité à son égard ${ }^{17}$. "

Pour comprendre les rapports qui unissent la population à ce personnel judiciaire présent sur le terrain, il faudrait également prendre en compte le nombre d'années durant lequel ils exercent leurs fonctions au sein d'un même lieu. En effet, les éventuelles collusions ne s'expliquent pas seulement par une origine géographique proche. Des rapports quotidiens facilitent la création de liens unissant des employés des Fermes, appâtés par une rémunération complémentaire s'ils ferment les yeux sur certains trafics, et la population attirée par les profits engendrés par la contrebande de tabac.

Dans le cadre d'une procédure criminelle, la collaboration entre les représentants de l'ordre et la population littorale existe également. Dès lors, il s'agit de nous demander qui fait appel à la justice. Ce sont surtout les notables et les nobles qui apparaissent dans les procès-verbaux. Ils sont perçus par la communauté comme de possibles intermédiaires : ils parlent français (ce qui n'est pas le cas de tous les habitants du littoral), ils savent écrire, donc cela évite les déplacements, ils sont impliqués dans la vie locale et deviennent par conséquent des porte-paroles. Cependant, ces notables n'agissent pas toujours au nom de la communauté urbaine ou rurale, mais parfois, pour leurs intérêts propres.

Certains personnages apparaissent systématiquement dans les procèsverbaux, tel le sieur Michel (dont nous ne connaissons pas la profession) à Quéven, qui est à l'origine de toutes les demandes d'interventions de la maréchaussée dans sa paroisse, soit cinq interventions entre janvier et mars 1785. Un autre personnage requiert souvent l'intervention de la justice : il s'agit du curé, qui, lui aussi à l'instar des notables, joue le rôle d'un intermédiaire. Il contacte les autorités. Par exemple, en cas de naufrages, il fait appel au personnel de l'amirauté mais il règle également les conflits entre cette juridiction et les habitants de sa paroisse : il réclame les salaires des gardiens et travailleurs commis au bris ou demande une récompense pour ses paroissiens venus d'eux-mêmes lui rapporter les effets retrouvés sur la grève comme le souligne Sylvain Coindet ${ }^{18}$.

16. Ibidem, p. 630 .

17. Ibid., p. 630-631.

18. COINDET, Sylvain, Les paroisses littorales face aux naufrages dans l'amirauté de Cornouaille, 1721/1740-1770/1790, mémoire de maîtrise rédigé sous la direction de Gérard LE BOUEDEC, UBS Lorient, oct. 2001, 201 p., voir p. 56 et p. 45. 
Nous savons peu de chose sur les autres personnes qui demandent l'intervention de la justice. Parfois, seule la mention " averti par la voie publique " apparaît dans les procès-verbaux rédigés par le personnel de la maréchaussée ou de l'amirauté. Dans certains cas, nous avons plus d'informations comme dans ce procès-verbal datant du 3 juillet 1787 rédigé par les cavaliers de maréchaussée de Vannes qui doivent agir :

"Sur ce que le nommé Louis Mendroux menuisier demeurant rue du moulin à Nantes, nous a requis de lui prester main forte à l'effet d'arrester une femme qui lui a volé un drap de lit et un mantelet suivant ce qu'il s'est apperçu rentrant de son travail et ignorer s'il ne lui manque pas d'autres effets, qu'en conséquence il a poursuivy la ditte femme à Paimboeuf, Donge, La Roche Bernard et est parvenu à la rencontrer dans cette ville rue du four dans l'auberge du nommé Dieux $[\ldots]^{19}$."

Malheureusement pour Louis Mendroux, qui a réussi au cours d'une longue poursuite à retrouver sa voleuse, les effets dérobés ont déjà été revendus. Ce n'est pas un cas isolé : on remarque en effet que la population n'hésite pas à avertir la justice voire à collaborer avec elle. Quand il s'agit d'un vol, cette collaboration est quasi-systématique car la propriété est une valeur fondamentale sous l'Ancien Régime. De même, les populations ne sont pas totalement ignorantes des rouages de la machine judiciaire : elles n'hésitent pas à déclencher une procédure ou au contraire à manipuler la justice dans le cadre de faux témoignages. Par contre, nous ne trouvons aucune demande d'intervention adressée aux employés des Fermes. Il est vrai que certaines juridictions sont plutôt mal perçues par la population car seule leur activité répressive apparaît, ce qui n'est, semblet-il, pas le cas pour l'amirauté ou les juridictions royales ordinaires. En effet, leur activité de services rendus à la population rend leur présence acceptable ou du moins tolérable sur le littoral.

Dès lors, mise à part l'indifférence, le second comportement adopté par les populations littorales est le refus voire le recours à la violence face aux représentants de l'autorité et à leur intrusion dans la vie quotidienne des communautés. Ce sont certainement les employés des Fermes qui pâtissent le plus de ces violences. Bon nombre d'arrestations de contrebandiers dégénèrent en révolte lorsque les habitants d'une paroisse littorale apprennent que l'un des leurs a été appréhendé. Le 13 août 1787, près d'Arzon, une charrette transportant une barrique apparaît comme suspecte aux commis :

"Nous avons sommé le conducteur de nous dire et déclarer ce qu'il portoit dans sa voiture et à l'instant, nous nous sommes retrouvés environnés d'une quantité d'hommes à nous inconnus armés de massues, de bâtons et qui se sont jettés à force ouverte sur nous, ont saisi le fusil de l'un de nous Dufour dont ils ont porté un coup sur la tête qui l'a renversé et laissé pour mort $^{20}$."

19. Arch. dép. du Morbihan, B 1334, affaire Marie Monnier.

20. Arch. dép. du Morbihan, 14 B 23. 
À Groix, en 1788, l'arrestation de trois hommes tourne mal et la population se révolte contre les commis dont l'un, nommé Faulard, sera la principale victime; son procès-verbal témoigne de la fureur de la population : " Nous Faulard ayant l'oreille à moitié coupée d'un coup de couteau de chasse, la tête et le corps mutilé $[\ldots]^{21}$."

L'intervention des employés des Fermes est très mal vécue par la population. Le tabac est, en effet, considéré comme un produit consommé par plaisir et les individus s'accommodent mal des réglementations le concernant. D'autant que les employés des Fermes prennent parfois leur mission très à cœur lorsqu'ils effectuent leurs fouilles : la requête du sieur Michel et de son épouse du 24 mars 1785 en témoigne :

" [...] Disant que parmi les excès auxquels se sont livré depuis l'établissement d'une franchise à Lorient, les commis des fermes du roi préposé à la conservation des droits à la barrière de cette ville, il n'est pas de plus répréhensibles que ceux dont le suppliant est fondé à se plaindre [...] Arrivé près de la barrière le sieur Lantier (capitaine général des Fermes) se lance vers la demoiselle Michel, la saisit par l'ouverture de sa juppe et crie aux employés arrestés moi cette saloppe là [...] ajoute aux employés : fouillez là, fouillez là et en même tems, il lui donne des coups de pieds dans le devant des jambes, dans les cuisses et dans le bas ventre de la demoiselle Michel qui est enceinte de deux mois [...] les autres employés la fouillèrent de la manière la plus indécente et la plus odieuse, ils [...] levaient ses hardes à lui découvrir les genoux et une partie des cuisses, avançaient même leurs mains hardies et impures jusque sous les nudités les plus cachées $[\ldots]^{22}$."

Les cavaliers de maréchaussée et les chasse-gueux subissent également les violences de la population lorsque leurs interventions dépassent les limites de l'admissible pour la communauté. Il existe un seuil de l'acceptable chez les populations littorales. Lorsque celui-ci est dépassé, elles réagissent par le refus de coopérer, la résistance ou le recours à la violence.

\section{Le regard porté par les populations sur l'efficacité des instances judiciaires}

Afin d'analyser le regard porté par les populations sur les instances judiciaires, nous utiliserons deux exemples : deux mémoires rédigés par des notables et les cahiers de doléances de 1789 des paroisses littorales.

Les notables considèrent la présence des représentants de l'autorité royale comme nécessaire et sollicitent même une augmentation des effectifs, car ceux-ci veillent sur leurs intérêts et sont une force de dissuasion contre le vol et le désordre. Le premier mémoire a été rédigé par les armateurs et les négociants de Nantes et envoyé au Parlement de Bretagne, le 24 mars 1774, au sujet de la brigade de maréchaussée créée à Paimboeuf quatre ans auparavant :

21. Arch. dép. du Morbihan, 14 B 23.

22. Arch. dép. du Morbihan, 14 B 22. 
"Les armateurs et négocians de Nantes prennent la liberté de représenter très humblement à Nosseigneurs du Parlement de Bretagne, qu'il est d'une conséquence infinie pour la marine marchande de faire exercer la plus exacte police dans les ports de mer : et que le moindre relâchement à ce sujet occasionneroit des vols et des désordres et la ruine des personnes attachées à la construction et à l'armement des vaisseaux [...] celui de Paimbœuf étant le plus exposé qu'aucun autre à ces enlèvements à cause de l'éloignement des armateurs, négocians et officiers de navires qui presque tous résident à Nantes : cette considération obligea le commerce à solliciter l'établissement d'une brigade de maréchaussée à Paimbœuf pour contenir le grand nombre de matelots, tant français qu'étrangers les charpentiers, calfats et gens de peine ${ }^{23}$."

Il est intéressant de noter l'image des populations flottantes véhiculée chez les élites comme étant indociles et réfractaires à toute forme d'autorité.

Le second mémoire a été vraisemblablement rédigé vers 1773 et insiste également sur la nécessité de la présence de la maréchaussée :

" La ville de L'Orient est celle de Bretagne où une brigade de maréchaussée seroit la plus nécessaire. On sentit cette nécessité en 1770, on y mit une brigade qui pendant deux années qu'elle y a resté a rendu les plus grands services : on juge à propos en 1772 de transporter cette brigade à Hennebont croyant que L'Orient pouvoit s'en passer, vu que cette ville a toujours un bataillon en garnison depuis ce tems là on y a bien sentis la privation de la brigade de maréchaussée, que d'assinats [sic], de vols, de désordres de toutes espèces ne se sont-ils pas commis ${ }^{24}$."

Ici encore, la suite du mémoire pointe du doigt les mauvais sujets qui résident dans le port et les gens de mer :

"L'Orient étant le lieu des embarquements pour l'Inde, il est celui où aboutissent tous les mauvais sujets du royaume qui veulent s'expatrier en se cachant sur les vaisseaux [...] L'Orient, comme tous les ports de mer, est remplis de matelots de toutes les nations, qui est l'espèce d'homme la plus difficile à contenir à terre. Depuis la cession de la Compagnie des Indes, la misère y est si grande qu'une quantité prodigieuse de gens qui n'ont plus les moyens de subsister s'adonne au brigandage [...] le tems de la vente des marchandises y attire une quantité prodigieuse de filous $[\ldots]^{25}$."

La situation est telle que la ville propose d'allouer la somme de 300 livres à l'officier qui prendra le commandement de la brigade pour le dédommager de la cherté des logements. Cependant, il s'agit là encore de la vision des notables et non pas de la population.

Les cahiers de doléances permettent également d'analyser la vision des populations littorales face aux pouvoirs. L'enchevêtrement des juridictions est mis en exergue par les populations : les habitants de Douarnenez demandent " que les tribunaux des seigneurs soient supprimés pour être réunis aux tribunaux supérieurs les plus prochains ${ }^{26}$ ". De même, la lon-

23. Arch. dép. de Loire-Atlantique, C 809, n 63.

24. Arch. dép. d'Ille-et-Vilaine, C 1135.

25. Arch. dép. d'Ille-et-Vilaine, C 1135.

26. Arch. dép. du Finistère, 10 B, cahier de doléances de Douarnenez 
gueur de la procédure pose problème : «Qu'il n'y ait à l'avenir que deux degrés de juridiction, et une supérieure en dernier ressort; que dans la première, faute aux juges de rendre dans l'an un jugement, les parties plaidantes ou l'une d'elles seront autorisées à se porter appelantes à la cour supérieure comme d'un déni de justice ${ }^{27}$. " La suppression des juridictions d'attribution est également une des requêtes présentées.

Les employés de la Ferme des Devoirs sont mal perçus. Ces doléances ne sont que le reflet de la mauvaise réputation dont ils jouissent tout au long de l'Ancien Régime :

« La classe la plus indigente est forcée de payer ses boissons pour ainsi dire le triple de la noblesse, du clergé, et d'autres personnes privilégiées par leur état. Le seul parti de procurer l'égalité est de supprimer la ferme des devoirs; un droit d'entrée sur chaque espèce de boissons procurerait à la province une indemnité équivalente à la ferme des devoirs, et rendrait à l'État un nombre infini d'individus jusqu'à ce jour inutiles ${ }^{28}$."

Jacques Péret fait la même remarque concernant les habitants de l'Aunis et de la Saintonge face aux employés des Fermes : "Dix-huit cahiers se lancent dans une attaque frontale contre cette "armée oppressive". Ces individus parlent également de "vexations" et de la "tyrannie fiscale ${ }^{29}$." " L'amirauté n'apparaît pas en tant que telle dans ces cahiers. Tout au plus se contente-t-on de l'assimiler aux juridictions d'attribution sans en faire une victime particulièrement désignée de la vindicte populaire. Jacques Péret arrive aux mêmes conclusions d'une critique modérée de cette juridiction dans les cahiers de doléances. Peut-être son activité de services légitime-t-elle sa présence aux yeux de la population?

Certaines paroisses témoignent aussi d'une volonté de réformer l'arsenal des peines jugé trop sévère : "Que les lois criminelles soient réformées, les peines adoucies et qu'il soit donné un défenseur aux accusés ${ }^{30}$."

La spécificité du littoral réside dans l'enchevêtrement de juridictions présentes sur cet espace : certaines d'entre elles ne sont pas liées au caractère maritime du territoire et exercent leurs attributions sur l'ensemble du royaume. Mais leur superposition induit une lecture difficile pour les populations (même si elles n'ignorent pas le fonctionnement de la justice, ce qui prouve qu'une certain processus d'acculturation est amorcé). Dès lors, les représentants de l'autorité royale, dont la tâche n'est pas aisée car ils ont à contrôler une population particulièrement mobile (population migrante et flottante), doivent maintenir un fragile équilibre dans les rapports qu'ils

27. Arch. dép. du Finistère, 10 B, cahier de doléances de Quimperlé.

28. Arch. dép. du Finistère, 10 B, cahier de doléances de Guilers.

29. PERET, Jacques, "Une société littorale et les pouvoirs d'Ancien Régime. Le cas de l'Aunis et de la Saintonge à travers les cahiers de doléances de 1789 ", dans Pouvoirs et littoraux du $X V^{e}$ au $x x^{e}$ siècle... op. cit., p. 79.

30. LE Mene, Michel et Santrot, Marie-Hélène, (dir.), Cahiers de plaintes et doléances de Loire-Atlantique, 1789, texte intégral et commentaires, t. 1, Nantes, éd du Conseil Général de Loire-Atlantique, 1989, 414 p., voir p. 407. 
entretiennent avec les communautés. De même, les populations littorales définissent les limites de l'acceptable : dès lors que l'intrusion des autorités dans leur vie quotidienne devient insupportable, elles le font savoir en utilisant notamment la révolte ou le contournement de la loi. Il semble également que le caractère essentiellement répressif de certaines juridictions comme les Traites soit un motif de résistance pour les populations. La rédaction des cahiers de doléances témoigne des préoccupations des populations : elles désirent une justice plus proche, moins coûteuse et surtout plus équitable.

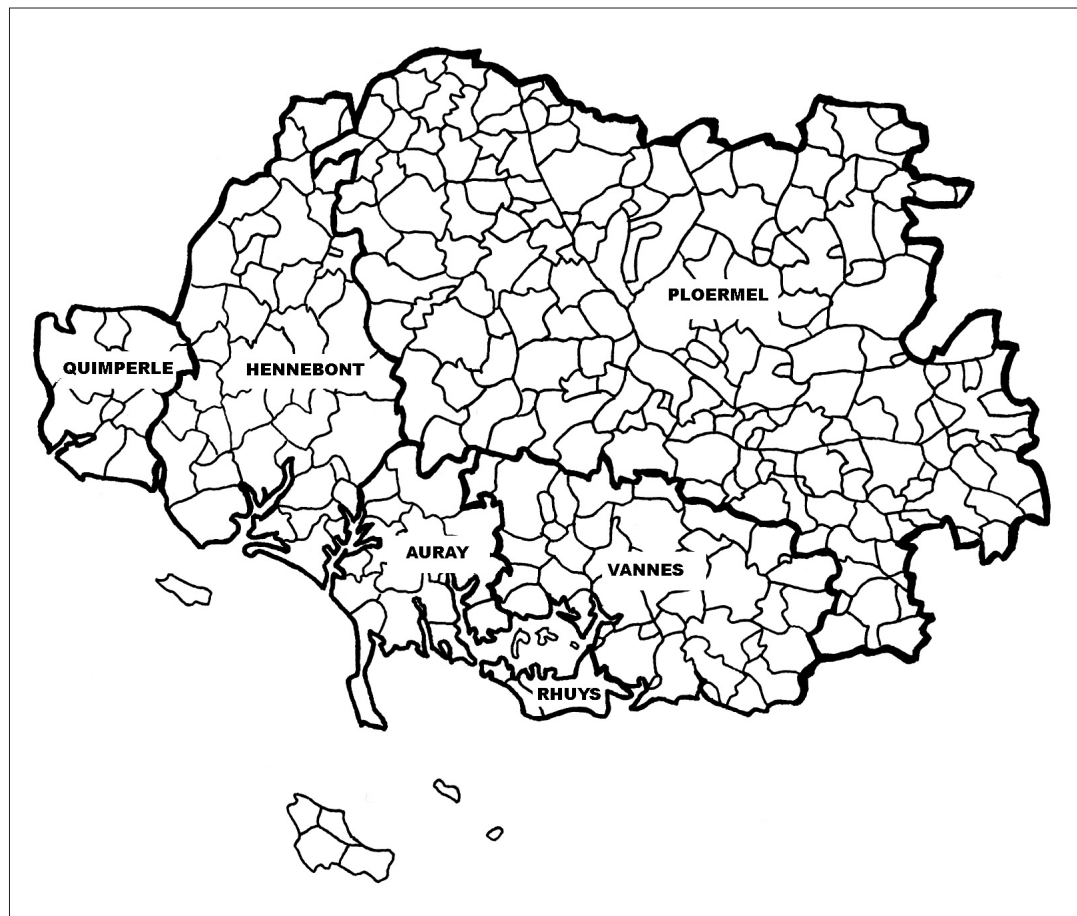


Figure 2-Le présidial de Nantes

et les sénéchaussées de son ressort en 1789

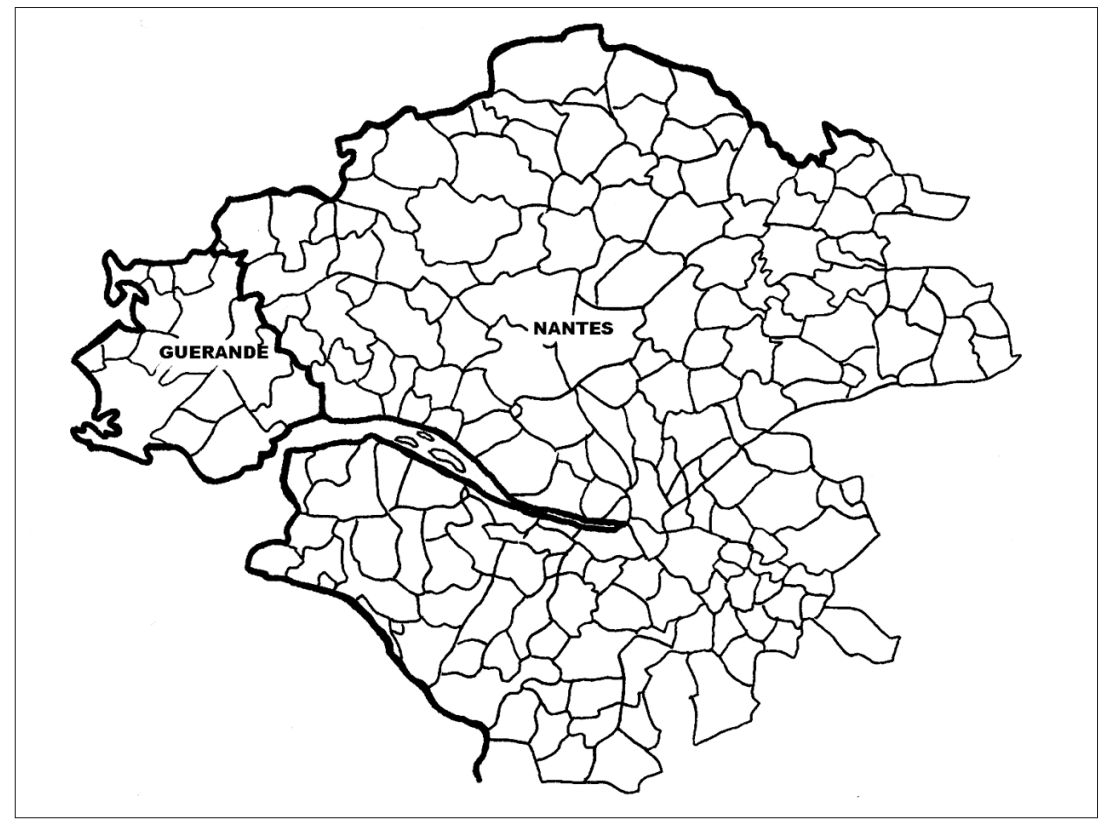

Figure 3 - Le présidial de Quimper

et les sénéchaussées de son ressort en 1789

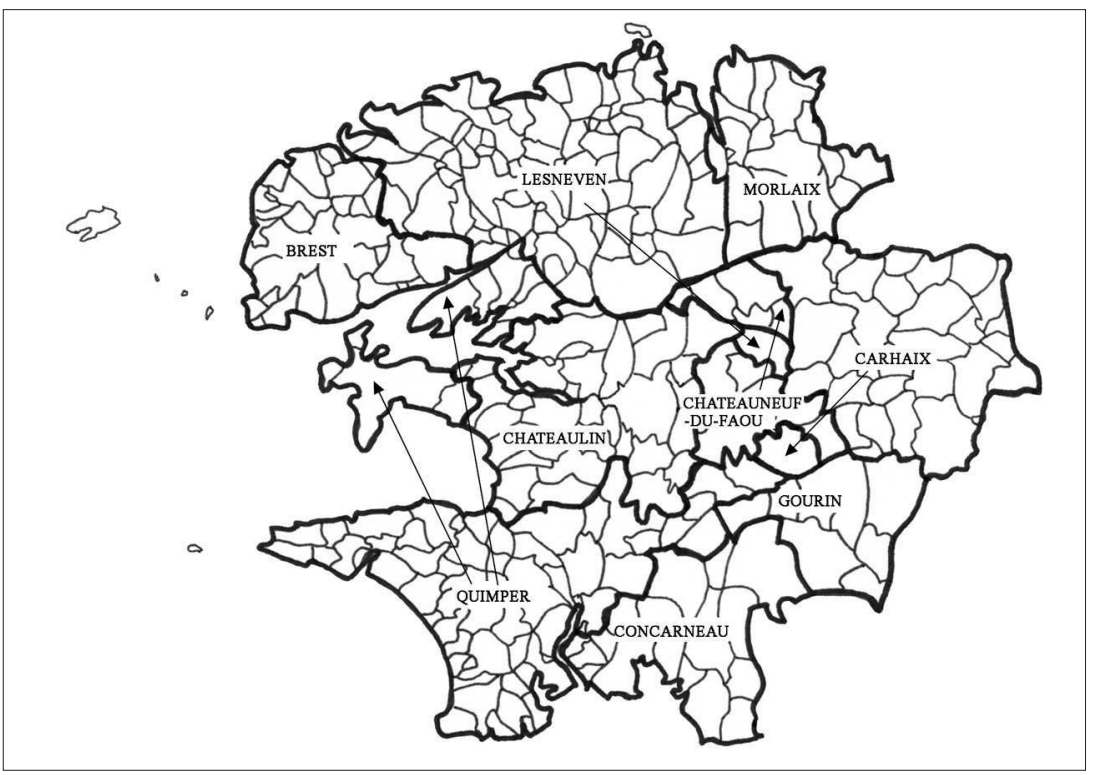




\section{RESUME}

L'encadrement de la population, une des principales obsessions de l'État sous l'Ancien Régime, a nécessité la constitution d'un maillage juridictionnel complexe et dense du territoire. Le littoral de la Bretagne méridionale et occidentale au $\mathrm{XVIII}^{\mathrm{e}}$ siècle apparaît comme un bon observatoire des rapports entre les habitants de cet espace et les représentants de l'ordre. Certaines juridictions peinent à faire reconnaître leur légitimité auprès des instances plus anciennes, car elles sont perçues comme des concurrentes potentielles. Il en résulte un manque d'efficacité dans la coordination des interventions et dans le suivi des affaires. De plus, cette juxtaposition des institutions judiciaires induit une lecture confuse chez les populations littorales et engendre un éventail de comportements, allant de la franche collaboration à la résistance la plus acharnée.

\section{ABSTRACT}

The supervision of the population, one of the main obsessions of the State during the Ancien Régime, required the creation of a complex and dense jurisdictional network on French territory. The southern and western coast of Brittany during the eighteenth century seems to be a good place in which one might make some observations to analyse the relationship between the people who lived in the area and State officials.

Some jurisdictions had to struggle to be recognized by older ones, because they were perceived as potential rivals. The result was a lack of efficiency to coordinate interventions and to follow through with cases. This juxtaposition of judicial institutions caused the coastal people to interpret it in a confused way, therefore creating a whole range of different behaviours, from open collaboration to the fiercest resistance. 